Severely immunocompromised patient are at high risk of infections caused by mould and fungi that can be dispersed into the hospital environment during construction and renovation activities. The most common fungal infection in these patients is Aspergillus and the associated mortality is between $60-90 \%$. Aspergillus is an environmental fungi that can be found in soil, water, decaying vegetation and the air. An increased level of dust and fungal spores during construction and renovation activities in healthcare settings has been associated with fungal infections in immunocompromised patients.

To minimise the risk of fungal infection and contamination of the healthcare environment during demolition, excavation and construction phases of the build, infection control risk assessments must be undertaken prior to commencement and as needed during the build. Such risk assessments document the type of build, the risk category in relation to patients and departments and the class of barriers required throughout each phase of the works.

Major construction and/or renovation activities require the establishment of a multidisciplinary team including but not limited to the healthcare facility major projects manager, hospitals engineer, infection prevention and control, clinical and department managers, environmental services manager, and architects and contracted building supervisors.

\section{HOSPITAL CLEANING: DETERGENT OR DISINFECTANT}

Stephanie Dancer

Hairmyres Hospital, East Kilbride, Lanarkshire, Scotland

The role of the healthcare environment in infection prevention and control has become increasingly important over the last decade. Pathogen reservoirs in hospitals have been linked with hospitalacquired infection (HAI) in both routine and outbreak settings. However, there remains debate over the delivery of cleaning services in a hospital, mainly due to lack of evidence. One of the most contentious issues is whether to use detergents or disinfectants for routine cleaning. This presentation will present evidence supporting the benefits of cleaning in reducing HAIs and compare and contrast data from studies using a range of different cleaning methods. Whichever method is chosen, the efficacy of a cleaning or disinfectant agent remains dependent upon physical action.

\section{HOW CAN ANTIBIOTICS MAKE US SICK AND WHAT TO DO ABOUT IT}

\section{Stephanie Dancer}

Hairmyres Hospital, East Kilbride, Lanarkshire, Scotland

Antibiotics were one of the most important discoveries last century and there is no doubt that they have revolutionised the treatment of infection. Unfortunately, these drugs can cause adverse effects that sometimes make things worse. One particular problem is the overgrowth of resistant organisms following antibiotic therapy. These so-called super-infections are often more difficult to treat because they are resistant; they will also spread to other patients if given the opportunity. Another problem is the fact that the original pathogen may be able to survive antibiotic treatment, so that an acute infection turns into a chronic one. It is even possible that exposure to antibiotics actually encourages the original pathogen to become more virulent. This presentation examines the evidence that giving a patient the 'wrong' antibiotic doesn't just fail to cure that patient; it results in a persistent infection with a more resistant pathogen, which then spreads to others and ultimately threatens the future management of infection. Better prescribing would help to minimise increasing antimicrobial resistance and this includes the courage to say 'no' to a request for antimicrobial agents. If a patient is systemically well, the benefits of treatment for the individual should be weighed against safeguarding treatment options for future patients.

\section{EBOLA VIRUSES: THE CHALLENGES OF DEVELOPING AND IMPLEMENTING DIAGNOSTICS FOR DANGEROUS EMERGING PATHOGENS}

\author{
David W. Smith ${ }^{1,2}$ \\ ${ }^{1}$ Department of Microbiology, PathWest Laboratory Medicine \\ WA, and ${ }^{2}$ School of Pathology and Laboratory Medicine, \\ University of Western Australia, Perth, WA, Australia
}

Ebolaviruses are members of the viral haemorrhagic fever virus group. They comprise five species, four of which are serious human pathogens and three of which have caused outbreaks of human disease in Africa. The ongoing outbreak in West Africa had almost 30,000 cases and over 11,000 deaths by mid-November 2015. This is the largest outbreak and the first in West Africa, and is caused by EBOV Zaire (EBOV) previously only known in central Africa. While the spread of these viruses within Australia is very unlikely, we have to be able to deal safely with potential cases in people entering Australia. Fundamental to that is the ability to provide timely and accurate diagnostic testing. That poses a number of interesting challenges because of the containment requirements for patients, specimens and the virus; the lack of clinical material for test development and validation; and the high personal and public health expectations for accurate diagnosis. Innovative approaches were needed to achieve this and provide the ongoing quality assurance required, but more work is needed to improve our capacity to deal with future outbreaks. Further challenges are faced in developing quality diagnostic testing capacity within West Africa.

\section{CURRENT STATUS OF EBOLAVIRUS VACCINE DEVELOPMENT}

\author{
Michael D. Nissen $^{1,2,3}$ \\ ${ }^{1}$ Director of Scientific Affairs and Public Health - Asia Pacific \\ and North Asia, GSK Vaccines Value and Health Science, \\ Singapore; ${ }^{2}$ GSK Vaccines, Wavre, Belgium; and ${ }^{3}$ Department \\ of Molecular and Cellular Pathology, School of Medicine, \\ Biomedical Sciences Faculty, University of Queensland, \\ St Lucia, Qld, Australia
}

The current Ebolavirus (EBOV) outbreak in West Africa remains uncontrolled, partially because there are still no approved vaccines or therapeutics to prevent or treat these infections. In the decade prior to 2014, many EBOV vaccines candidates had been identified, though none had progressed to registration for clinical use in humans. This latest and largest EBOV outbeak has renewed the urgency for an effective and safe vaccine strategy to combat further epidemics. Several EBOV vaccine candidates have now advanced to human clinical trials. These vaccines are based on their ability to elicit immune responses against EBOV glycoproteins (GPs; the major viral immunogens): DNA-based 